\title{
Three dimensional surfaces foliated by the equiform motion of pseudohyperbolic surfaces in $\mathrm{E}^{7}$
}

\author{
E. M. Solouma ${ }^{1}$
}

Department of Mathematics and Statistics, College of Science, Al Imam Muhammad Ibn Saud Islamic University, Kingdom of Saudi Arabia E-mail: emadms74@yahoo.com.

\section{Abstract}

Department of Mathematics, College of Science, Beni-Suef University, Beni-Suef, Egypt

In this paper we study three dimensional surfaces in $E^{7}$ generated by equiform motions of a pseudohyperbolic surface. The properties of these surfaces up to the first order are investigated. We prove that three dimensional surfaces in $E^{7}$ in general, is contained in a canal hypersurface, which is gained as envelope of a one-parametric set of 6 -dimensional pseudohyperbolic. Finally we give an example.

Mathematical Subject Classification (2010): 53A05, 53A17, 53B30.

Keywords: Equiform motion; hypersurfaces; Lorentz metrics.

\section{Council for Innovative Research}

Peer Review Research Publishing System

Journal: INTERNATIONAL JOURNAL OF COMPUTERS \& TECHNOLOGY

Vol 12, No. 9

· editor@cirworld.com

www.cirworld.com, www.ijctonline.com 


\section{Introduction}

An equiform transformation in the $n$-dimensional Euclidean space $\mathrm{R}^{n}$ is an affine transformation whose linear part is composed from an orthogonal transformation and a homothetical transformation. Such an equiform transformation maps points $\mathbf{x} \in \mathrm{R}^{n}$ according to

$$
\mathbf{x} \mapsto s A \mathbf{x}+\mathbf{d}, \quad .5 c m A \in S O(n), s \in \mathrm{R}^{+}, \mathbf{d} \in \mathrm{R}^{n} .
$$

The number $s$ is called the scaling factor. An equiform motion is defined if the parameters of (1), including $s$, are given as functions of a time parameter $t$. Then a smooth one-parameter equiform motion moves a point $\mathbf{X}$ via $\mathbf{x}(t)=s(t) A(t) \mathbf{x}(t)+\mathbf{d}(t)$. The kinematic corresponding to this transformation group is called equiform kinematic. See [2]. Recently, the equiform kinematic geometry has been used in computer vision and reverse engineering of geometric models such as the problem of reconstruction of a computer model from an existing object which is known (a large number of) data points on the surface of the technical object [9, 11]. In [8], they studied two-parameter spatial motions $M_{2}(\lambda, \mu)$ in three dimensional Euclidean space from a differential geometric point of view, which (up to the second order) instantaneously move on locally one-dimensional point paths. In [1, 12], they studied some first order properties of cyclic surfaces generated by the equiform motions in five dimensional Euclidian space and semi-Euclidean space.

In Minkowski (semi-Euclidean) space $\mathrm{E}^{3}$ with scalar product $\langle x, y\rangle=-x_{1} y_{1}+x_{2} y_{2}+x_{3} y_{3}$ the pseudosphere or Lorentz sphere and the pseudohyperbolic surface play the same role as sphere in Euclidean space. Lorentz sphere of radius $r>0$ in $\mathrm{E}^{3}$ is the quadric

$$
S^{2}(r)=\left\{p \in \mathrm{E}^{3}:<p, p>=r^{2}\right\} .
$$

This surface is timelike and is the hyperboloid of one sheet $-x_{1}^{2}+x_{2}^{2}+x_{3}^{2}=r^{2}$ which is obtained by rotating the hyperbola $-x_{1}^{2}+x_{3}^{2}=r^{2}$ in the plane $x_{2}=0$ with respect to the $x_{1}$-axis. The pseudohyperbolic surface is the quadratic

$$
H_{0}^{2}(r)=\left\{p \in \mathrm{E}^{3}:<p, p>=-r^{2}\right\}
$$

This surface is spacelike and is the hyperboloid of two sheet $-x_{1}^{2}+x_{2}^{2}+x_{3}^{2}=-r^{2}$ which is obtained by rotating the hyperbola $x_{1}^{2}-x_{3}^{2}=r^{2}$ in the plane $x_{2}=0$ with respect to the $x_{1}$-axis [10].

In this paper we consider the equiform motions of a pseudohyperbolic surface $k_{\circ}$ in $\mathrm{E}^{n}$. The point paths of the pseudohyperbolic surface, generate three-dimensional surface, contains the positions of the starting pseudohyperbolic surface $k_{\circ}$. The first order properties of these surfaces for the points of these pseudohyperbolic surfaces have been studied for arbitrary dimensions $n \geq 3$. We restrict our considerations to dimension $n=7$ because, at any moment the infinitesimal transformations of the motion maps the points of the pseudohyperbolic surface $k_{\circ}$ to the velocity vectors, whose end points will form an affine image of $k_{\circ}$ (in general a pseudohyperbolic surface $k_{\circ}$ ). Both these surfaces are space and therefore span a subspace $W$ of $\mathrm{E}^{n}$ with $n \leq 7$. Moreover, we show that any three-dimensional surfaces in $E^{7}$ is in general contained in a canal hypersurface, which is gained as envelope of a one-parametric set of 6-dimensional pseudosphere.

\section{Local study in canonical frames}

Consider a unit pseudohyperbolic surface $k_{\circ}$ in the space $\pi_{\circ}=\left[x_{1} x_{2} x_{3}\right]$ centered at the origin represented by

$$
x(\theta, \phi)=(\cosh \theta, \sinh \theta \sin \phi, \sinh \theta \cos \phi, 0,0,0,0)^{T}, \theta \in \mathrm{R} \text { and } \phi \in[0,2 \pi]
$$

the general representation of the motion of three-dimensional surface in $E^{7}$ foliated by two-dimensional pseudohyperbolic surface is given by 


$$
X(t, \theta, \phi)=s(t) A(t) x(\theta, \phi)+d(t), t \in \mathrm{R}
$$

where $d(t)=\left(b_{1}(t), b_{2}(t), b_{3}(t), b_{4}(t), b_{5}(t), b_{6}(t), b_{7}(t)\right)^{T}$ describes the position of the origin of $\Sigma^{\circ}$ at the time $t, A(t)=\left(a_{i j}(t)\right), 1 \leq i, j \leq 7$ is a semi orthogonal matrix and $s(t)$ provides the scaling factor of the moving system. Moreover we assume that all involved functions are of class $C^{1}$. Using Taylor's expansion, up to the first order then the representation of the motion is given by

$$
X(t, \theta, \phi)=\{s(0) A(0)+[\dot{s}(0) A(0)+s(0) \dot{A}(0)] t\} x(\theta, \phi)+d(0)+t \dot{d}(0),
$$

where $(\cdot)$ denotes differentiation with respect to time $(t=0)$. As an equiform motion has an invariant point, we can assume without loss of generality that the moving frame $E^{7}$ and fixed frame $\Sigma$ coinciding at the zero position $(t=0)$, then we have

$$
A(0)=I, \quad s(0)=1 \text { and } d(0)=0,
$$

thus

$$
X(t, \theta, \phi)=\left[I+\left(s^{\prime} I+\Omega\right) t\right] x(\theta, \phi)+t d^{\prime},
$$

where $\Omega=\dot{A}(0)=\left(\omega_{k}\right), k=1,2,3, \ldots, 21$ is a semi skew symmetric matrix. In this paper all values of $s, b_{i}$ and their derivatives are computed at $t=0$ and for simplicity, we write $s^{\prime}$ and $b_{i}^{\prime}$ instead of $\dot{s}(0)$ and $\dot{b}_{i}(0)$ respectively. In these frames, the representation of $\mathbf{X}(t, \theta, \phi)$ is given by

$\left(\begin{array}{l}X_{1} \\ X_{2} \\ X_{3} \\ X_{4} \\ X_{5} \\ X_{6} \\ X_{6}\end{array}\right)=\left(\begin{array}{cccccccc}1+s^{\prime} t & t \omega_{1} & t \omega_{2} & t \omega_{3} & t \omega_{4} & t \omega_{5} & t \omega_{6} \\ t \omega_{1} & 1+s^{\prime} t & t \omega_{7} & t \omega_{8} & t \omega_{9} & t \omega_{10} & t \omega_{11} \\ t \omega_{2} & -t \omega_{7} & 1+s^{\prime} t & t \omega_{12} & t \omega_{13} & t \omega_{14} & t \omega_{15} \\ t \omega_{3} & -t \omega_{8} & -t \omega_{12} & 1+s^{\prime} t & t \omega_{16} & t \omega_{17} & t \omega_{18} \\ t \omega_{4} & -t \omega_{9} & -t \omega_{13} & -t \omega_{16} & 1+s^{\prime} t & t \omega_{19} & t \omega_{20} \\ t \omega_{5} & -t \omega_{10} & -t \omega_{14} & -t \omega_{17} & -t \omega_{19} & 1+s^{\prime} t & t \omega_{21} \\ t \omega_{6} & -t \omega_{11} & -t \omega_{15} & -t \omega_{18} & -t \omega_{20} & -t \omega_{21} & 1+s^{\prime} t\end{array}\right)\left(\begin{array}{c}\cosh \phi \\ \sinh \theta \sin \phi \\ \sinh \theta \cos \phi \\ 0 \\ 0 \\ 0 \\ \text { or in the equivalent form }\end{array}\right)+t\left(\begin{array}{l}b_{1}^{\prime} \\ b_{2}^{\prime} \\ b_{3}^{\prime} \\ b_{4}^{\prime} \\ b_{5}^{\prime} \\ b_{6}^{\prime} \\ b_{7}^{\prime}\end{array}\right)$,

$$
\begin{aligned}
\left(\begin{array}{l}
X_{1} \\
X_{2} \\
X_{3} \\
X_{4} \\
X_{5} \\
X_{6} \\
X_{7}
\end{array}\right)=\left(\begin{array}{l}
b_{1}^{\prime} \\
b_{2}^{\prime} \\
b_{3}^{\prime} \\
b_{4}^{\prime} \\
b_{5}^{\prime} \\
b_{6}^{\prime} \\
b_{7}^{\prime} \\
t \omega_{2} \\
t \omega_{3} \\
t \omega_{4} \\
t \omega_{5} \\
t \omega^{\prime}
\end{array}\right)\left(\begin{array}{c}
t \omega_{1} \\
1+s^{\prime} t \\
-t \omega_{7} \\
-t \omega_{8} \\
-t \omega_{9} \\
-t \omega_{10} \\
-t \omega_{11}
\end{array}\right) \sinh \theta \sin \phi+\left(\begin{array}{c}
t \omega_{2} \\
t \omega_{7} \\
1+s^{\prime} t \\
-t \omega_{12} \\
-t \omega_{13} \\
-t \omega_{14} \\
-t \omega_{15}
\end{array}\right) \sinh \theta \cos \phi \\
\\
=t \vec{b}+\vec{a}_{0} \cosh \theta+\vec{a}_{1} \sinh \theta \sin \phi+\vec{a}_{2} \sinh \theta \cos \phi .
\end{aligned}
$$


For any fixed $t$ in the above expression (3), we generally gain an elliptical hyperboloid for $\theta \in \mathrm{R}$ and $\phi \in[0,2 \pi]$ centered at the point $t\left(b_{1}^{\prime}, b_{2}^{\prime}, b_{3}^{\prime}, b_{4}^{\prime}, b_{5}^{\prime}, b_{6}^{\prime}, b_{7}^{\prime}\right)$. The latter elliptical hyperboloid turns to a two-dimensional pseudohyperbolic surface if $\vec{a}_{0}, \vec{a}_{1}$ and $\vec{a}_{2}$ form an orthogonal basis. This gives the conditions

$$
\begin{aligned}
\omega_{2} \omega_{7}+\omega_{3} \omega_{8}+\omega_{4} \omega_{9}+\omega_{5} \omega_{10}+\omega_{6} \omega_{11} & =-\omega_{1} \omega_{7}+\omega_{3} \omega_{12}+\omega_{4} \omega_{13}+\omega_{5} \omega_{14}+\omega_{6} \omega_{15} \\
& =-\omega_{1} \omega_{2}+\omega_{8} \omega_{12}+\omega_{9} \omega_{13}+\omega_{10} \omega_{14}+\omega_{11} \omega_{15} \\
& =0
\end{aligned}
$$

and

$$
\begin{aligned}
\omega_{1}^{2}+\omega_{2}^{2}+\omega_{3}^{2}+\omega_{4}^{2}+\omega_{5}^{2}+\omega_{6}^{2} & =\omega_{1}^{2}-\omega_{7}^{2}-\omega_{8}^{2}-\omega_{9}^{2}-\omega_{10}^{2}-\omega_{11}^{2} \\
& =\omega_{2}^{2}-\omega_{7}^{2}-\omega_{12}^{2}-\omega_{13}^{2}-\omega_{14}^{2}-\omega_{15}^{2} \\
& =a,
\end{aligned}
$$

where $a \in \mathrm{R}^{+}$. Thus we get the following equation of the pseudohyperbolic space

$$
\sum_{i=1}^{7} \varepsilon_{i}\left(x_{i}-t b_{i}^{\prime}\right)^{2}=a t^{2}-\left(1+s^{\prime} t\right)^{2}
$$

where $\varepsilon_{1}=-1, \varepsilon_{j}=1, j=2,3,4,5,6,7$. The orthogonal projection of these elliptical hyperboloid $(t=$ const . in (3)) on the space of the starting pseudohyperbolic surface $\pi_{\circ}=\left[x_{1} x_{2} x_{3}\right]$, is

$$
\left(\begin{array}{l}
X_{1} \\
X_{2} \\
X_{3}
\end{array}\right)=t\left(\begin{array}{l}
b_{1}^{\prime} \\
b_{2}^{\prime} \\
b_{3}^{\prime}
\end{array}\right)+\left(\begin{array}{c}
1+s^{\prime} t \\
t \omega_{1} \\
t \omega_{2}
\end{array}\right) \cosh \theta+\left(\begin{array}{c}
t \omega_{1} \\
1+s^{\prime} t \\
-t \omega_{7}
\end{array}\right) \sinh \theta \sin \phi+\left(\begin{array}{c}
t \omega_{2} \\
t \omega_{7} \\
1+s^{\prime} t
\end{array}\right) \sinh \theta \cos \phi
$$

This equation generalizes in five dimension that happens for $\phi=0$. Namely, if $\phi=0$ the orthogonal projection of the elliptical hyperboloid in equation (4) on the space $\left[x_{1} x_{3}\right]$ is

$$
\left(\begin{array}{l}
X_{1} \\
X_{3}
\end{array}\right)=t\left(\begin{array}{l}
b_{1}^{\prime} \\
b_{3}^{\prime}
\end{array}\right)+\left(\begin{array}{c}
1+s^{\prime} t \\
t \omega_{2}
\end{array}\right) \cosh \theta+\left(\begin{array}{c}
t \omega_{2} \\
1+s^{\prime} t
\end{array}\right) \sinh \theta
$$

This gives Lorentzian circles centered at $\left(t b_{1}^{\prime}, t b_{3}^{\prime}\right)$ and radii by $\sqrt{\left|t^{2} \omega_{2}^{2}-\left(1+s^{\prime} t\right)^{2}\right|}$.

\section{Corollary 2.1}

The projection of the ruled surface of tangent to $k_{\circ}$ into the original space will give a three-dimensional surface in $\mathrm{E}^{3}$ , which is foliated by elliptical hyperboloids. Now from (4) we have

$$
X(t, \theta, \phi)=\left(\begin{array}{ccc}
1+s^{\prime} t & t \omega_{1} & t \omega_{2} \\
t \omega_{1} & 1+s^{\prime} t & t \omega_{7} \\
t \omega_{2} & -t \omega_{7} & 1+s^{\prime} t
\end{array}\right)\left(\begin{array}{c}
\cosh \theta \\
\sinh \theta \sin \phi \\
\sinh \theta \cos \phi
\end{array}\right)+t\left(\begin{array}{l}
b_{1}^{\prime} \\
b_{2}^{\prime} \\
b_{3}^{\prime}
\end{array}\right),
$$


and the first partial derivatives are

$$
\begin{aligned}
X_{t} & =\left(\begin{array}{l}
b_{1}^{\prime} \\
b_{2}^{\prime} \\
b_{3}^{\prime}
\end{array}\right)+\left(\begin{array}{ccc}
s^{\prime} & \omega_{1} & \omega_{2} \\
\omega_{1} & s^{\prime} & \omega_{7} \\
\omega_{2} & -\omega_{7} & s^{\prime}
\end{array}\right)\left(\begin{array}{c}
\cosh \theta \\
\sinh \theta \sin \phi \\
\sinh \theta \cos \phi
\end{array}\right), \\
X_{\theta} & =(\sinh \theta, \cosh \theta \sin \phi, \cosh \theta \cos \phi)^{T}, \\
X_{\phi} & =(0, \sinh \theta \cos \phi,-\sinh \theta \sin \phi)^{T} .
\end{aligned}
$$

Then the linearly dependent points

$$
\sinh \theta\left[-s^{\prime}-b_{1}^{\prime} \cosh \theta+b_{2}^{\prime} \sinh \theta \sin \phi+b_{3}^{\prime} \sinh \theta \cos \phi\right]=0,
$$

we get

$$
\sinh \theta\left[-s^{\prime}+<d^{\prime}, x(\theta, \phi)>\right]=0 .
$$

The latter equation characterizes the instantaneous curve of contact.

\section{Tangent pseudosphere of three-dimensional surface in $\mathrm{E}^{7}$}

In this section we will how that at any instant $t$ there exists a pseudosphere $K(t)$, which is tangent to a given three-dimensional surface (2) in all points of the instantaneous position $k(t)$ of the pseudohyperbolic surface $k_{\circ}$.

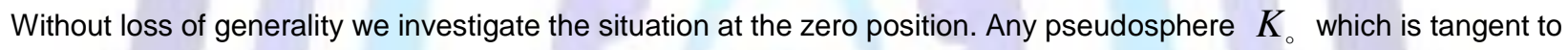
the given three-dimensional surface (2) along $k_{\circ}$ has to contain $k_{\circ}$, hence the center of $K_{\circ}$ has coordinates $\left(0,0,0, m_{4}, m_{5}, m_{6}, m_{7}\right)$ with $m_{4}, m_{5}, m_{6}, m_{7} \in \mathrm{R}$. On the other hand since $K_{\circ}$ has to be tangent to all velocity vectors of the motion, the center of $K$ 。 has to lie in each of the hyperplanes through the points of $k(t)$ orthogonal to these velocity vectors. This gives us the additional condition

$$
\begin{aligned}
& m_{4}\left(b_{4}^{\prime}+\omega_{3} \cosh \theta-\omega_{8} \sinh \theta \sin \phi-\omega_{12} \sinh \theta \cos \phi\right) \\
& +m_{5}\left(b_{5}^{\prime}+\omega_{4} \cosh \theta-\omega_{9} \sinh \theta \sin \phi-\omega_{13} \sinh \theta \cos \phi\right) \\
& +m_{6}\left(b_{6}^{\prime}+\omega_{5} \cosh \theta-\omega_{10} \sinh \theta \sin \phi-\omega_{14} \sinh \theta \cos \phi\right) \\
& +m_{7}\left(b_{7}^{\prime}+\omega_{6} \cosh \theta-\omega_{11} \sinh \theta \sin \phi-\omega_{15} \sinh \theta \cos \phi\right) \\
& =-s^{\prime}-b_{1}^{\prime} \cosh \theta+b_{2}^{\prime} \sinh \theta \sin \phi+b_{3}^{\prime} \sinh \theta \cos \phi
\end{aligned}
$$

By comparing the coefficients of $\{1, \cosh \theta, \sinh \theta \sin \phi, \sinh \theta \cos \phi\}$ in (5), we have the system of linear equations

$$
B M=H,
$$

where

$$
B=\left(\begin{array}{cccc}
b_{4}^{\prime} & b_{5}^{\prime} & b_{6}^{\prime} & b_{7}^{\prime} \\
\omega_{3} & \omega_{4} & \omega_{5} & \omega_{6} \\
\omega_{8} & \omega_{9} & \omega_{10} & \omega_{11} \\
\omega_{12} & \omega_{13} & \omega_{14} & \omega_{15}
\end{array}\right), \quad M=\left(\begin{array}{l}
m_{4} \\
m_{5} \\
m_{6} \\
m_{7}
\end{array}\right) \text { and } \quad H=\left(\begin{array}{c}
-s^{\prime} \\
-b_{1}^{\prime} \\
-b_{2}^{\prime} \\
-b_{3}^{\prime}
\end{array}\right)
$$

If $B$ is a regular matrix, we get 


$$
M=B^{-1} H
$$

Therefore, we have the following theorem:

Theorem 3.1

Definition 3.1 Canal hypersurfaces in $\mathrm{E}^{n}$ are envelope hypersurfaces of one-parametric sets of pseudospheres. Therefore, we have the following theorem

\section{Theorem 3.2}

\subsection{The singular cases}

If the system of equations (6) is singular, we have many cases:

Case 1. $\operatorname{rank}(B)=\operatorname{rank}(B \backslash H)=3$. In this case, we have a one-parametric set of pseudospheres whose centers fulfil a straight line in the $x_{4} x_{5} x_{6} x_{7}$-space

$$
M=\left(0,0,0, m_{4}, x_{5}\left(m_{4}\right), x_{6}\left(m_{4}\right), x_{7}\left(m_{4}\right)\right),
$$

where

$$
\begin{aligned}
x_{5}\left(m_{4}\right)= & \frac{1}{\Delta^{*}}\left[\left(\omega_{5} \omega_{11}-\omega_{6} \omega_{10}\right)\left(s^{\prime}+b_{4}^{\prime} m_{4}\right)+\left(b_{6}^{\prime} \omega_{11}-b_{7}^{\prime} \omega_{10}\right)\left(b_{1}^{\prime}+\omega_{3} m_{4}\right)\right. \\
& \left.+\left(b_{7}^{\prime} \omega_{5}-b_{6}^{\prime} \omega_{6}\right)\left(b_{2}^{\prime}+\omega_{8} m_{4}\right)\right], \\
x_{6}\left(m_{4}\right)= & \frac{1}{\Delta^{*}}\left[\left(\omega_{6} \omega_{9}-\omega_{4} \omega_{11}\right)\left(s^{\prime}+b_{4}^{\prime} m_{4}\right)+\left(b_{7}^{\prime} \omega_{9}-b_{5}^{\prime} \omega_{11}\right)\left(b_{1}^{\prime}+\omega_{3} m_{4}\right)\right. \\
& \left.+\left(b_{5}^{\prime} \omega_{6}-b_{7}^{\prime} \omega_{4}\right)\left(b_{2}^{\prime}+\omega_{8} m_{4}\right)\right], \\
x_{7}\left(m_{4}\right)= & \frac{1}{\Delta^{*}}\left[\left(\omega_{4} \omega_{10}-\omega_{5} \omega_{9}\right)\left(s^{\prime}+b_{4}^{\prime} m_{4}\right)+\left(b_{5}^{\prime} \omega_{10}-b_{6}^{\prime} \omega_{9}\right)\left(b_{1}^{\prime}+\omega_{3} m_{4}\right)\right. \\
& \left.+\left(b_{6}^{\prime} \omega_{4}-b_{5}^{\prime} \omega_{5}\right)\left(b_{2}^{\prime}+\omega_{8} m_{4}\right)\right],
\end{aligned}
$$

where

$$
\Delta^{*}=b_{5}^{\prime}\left(\omega_{5} \omega_{11}-\omega_{6} \omega_{9}\right)+b_{6}^{\prime}\left(\omega_{6} \omega_{9}-\omega_{4} \omega_{11}\right)+b_{7}^{\prime}\left(\omega_{4} \omega_{10}-\omega_{5} \omega_{9}\right),
$$

with arbitrary $m_{4} \in \mathrm{R}$. Thus, we get a straight line of possible centers.

Case 2. $\operatorname{rank}(B)=\operatorname{rank}(B \backslash H)=2$. In this case, we have a two-parametric set of pseudospheres whose centers fulfil a surface in $x_{4} x_{5} x_{6} x_{7}-$ space

$$
M=\left(0,0,0, m_{4}, m_{5}, x_{6}\left(m_{4}, m_{5}\right), x_{7}\left(m_{4}, m_{5}\right)\right),
$$

where

$$
x_{6}\left(m_{4}, m_{5}\right)=\frac{m_{4}\left(b_{6}^{\prime} \omega_{3}-b_{4}^{\prime} \omega_{5}\right)+m_{5}\left(b_{6}^{\prime} \omega_{4}-b_{5}^{\prime} \omega_{5}\right)+\left(s^{\prime} \omega_{5}-b_{1}^{\prime} b_{6}^{\prime}\right)}{b_{7}^{\prime} \omega_{5}-b_{6}^{\prime} \omega_{6}},
$$




$$
x_{7}\left(m_{4}, m_{5}\right)=\frac{m_{4}\left(b_{4}^{\prime} \omega_{6}-b_{7}^{\prime} \omega_{3}\right)+m_{5}\left(b_{5}^{\prime} \omega_{6}-b_{7}^{\prime} \omega_{4}\right)-\left(s^{\prime} \omega_{6}-b_{1}^{\prime} b_{7}^{\prime}\right)}{b_{7}^{\prime} \omega_{5}-b_{6}^{\prime} \omega_{6}},
$$

with arbitrary $m_{4}, m_{5} \in \mathrm{R}$. Thus, we get a surface of possible centers.

Case 3. $\operatorname{rank}(B)=\operatorname{rank}(B \backslash H)=1$. In this case, we have a hyperplane of possible centers. Case 4. $\operatorname{rank}(B)=3 \neq \operatorname{rank}(B \backslash H)$. In this case we assume

$$
\frac{\omega_{8}}{\omega_{12}}=\frac{\omega_{9}}{\omega_{13}}=\frac{\omega_{10}}{\omega_{14}}=\frac{\omega_{11}}{\omega_{15}}=\lambda, \quad \frac{b_{2}^{\prime}}{b_{3}^{\prime}} \neq \lambda .
$$

By using the homogenous coordinates

$$
\begin{gathered}
m_{\circ}=\Delta=0, \quad m_{1}=0, \quad m_{2}=0, \quad m_{3}=0 \\
m_{4}=\left(b_{2}^{\prime}-\lambda b_{3}^{\prime}\right)\left[b_{5}^{\prime}\left(\omega_{6} \omega_{14}-\omega_{5} \omega_{15}\right)+b_{6}^{\prime}\left(\omega_{4} \omega_{15}-\omega_{6} \omega_{13}\right)+b_{7}^{\prime}\left(\omega_{5} \omega_{13}-\omega_{4} \omega_{14}\right)\right] \\
m_{5}=\left(b_{2}^{\prime}-\lambda b_{3}^{\prime}\right)\left[b_{4}^{\prime}\left(\omega_{5} \omega_{15}-\omega_{6} \omega_{14}\right)+b_{6}^{\prime}\left(\omega_{6} \omega_{12}-\omega_{3} \omega_{15}\right)+b_{7}^{\prime}\left(\omega_{3} \omega_{14}\right)-\omega_{5} \omega_{12}\right] \\
m_{6}=\left(b_{2}^{\prime}-\lambda b_{3}^{\prime}\right)\left[b_{4}^{\prime}\left(\omega_{6} \omega_{13}-\omega_{4} \omega_{15}\right)+b_{5}^{\prime}\left(\omega_{3} \omega_{15}-\omega_{6} \omega_{12}\right)+b_{7}^{\prime}\left(\omega_{4} \omega_{12}-\omega_{3} \omega_{13}\right)\right] \\
m_{7}=\left(b_{2}^{\prime}-\lambda b_{3}^{\prime}\right)\left[b_{4}^{\prime}\left(\omega_{4} \omega_{14}-\omega_{5} \omega_{13}\right)+b_{5}^{\prime}\left(\omega_{5} \omega_{12}-\omega_{3} \omega_{14}\right)+b_{6}^{\prime}\left(\omega_{3} \omega_{13}-\omega_{4} \omega_{12}\right)\right]
\end{gathered}
$$

Then the centers of the pseudospheres are an ideal point (point at infinity). The corresponding pseudospheres degenerates into a hyperplane.

Case 5. $\operatorname{rank}(B)=2 \neq \operatorname{rank}(B \backslash H)$. In this case we assume

$$
\begin{aligned}
& \frac{\omega_{3}}{\omega_{8}}=\frac{\omega_{4}}{\omega_{9}}=\frac{\omega_{5}}{\omega_{10}}=\frac{\omega_{6}}{\omega_{11}}=\lambda, \quad \frac{b_{1}^{\prime}}{b_{2}^{\prime}} \neq \lambda, \\
& \frac{\omega_{8}}{\omega_{12}}=\frac{\omega_{9}}{\omega_{13}}=\frac{\omega_{10}}{\omega_{14}}=\frac{\omega_{11}}{\omega_{15}}=\mu, \quad \frac{b_{2}^{\prime}}{b_{3}^{\prime}} \neq \mu .
\end{aligned}
$$

Using the homogenous coordinates

$$
\begin{gathered}
m_{\circ}=\Delta=0, \quad m_{1}=0, \quad m_{2}=0, \quad m_{3}=0 \\
m_{4}=\left(\lambda b_{2}^{\prime}-\lambda \mu b_{3}^{\prime}\right)\left[b_{5}^{\prime}\left(\omega_{11} \omega_{14}-\omega_{10} \omega_{15}\right)+b_{6}^{\prime}\left(\omega_{9} \omega_{15}-\omega_{11} \omega_{13}\right)+b_{7}^{\prime}\left(\omega_{10} \omega_{13}-\omega_{9} \omega_{14}\right)\right] \\
m_{5}=\left(\lambda b_{2}^{\prime}-\lambda \mu b_{3}^{\prime}\right)\left[b_{4}^{\prime}\left(\omega_{10} \omega_{15}-\omega_{11} \omega_{14}\right)+b_{6}^{\prime}\left(\omega_{11} \omega_{12}-\omega_{8} \omega_{15}\right)+b_{7}^{\prime}\left(\omega_{8} \omega_{14}\right)-\omega_{10} \omega_{12}\right]
\end{gathered}
$$




$$
\begin{aligned}
& m_{6}=\left(\lambda b_{2}^{\prime}-\lambda \mu b_{3}^{\prime}\right)\left[b_{4}^{\prime}\left(\omega_{11} \omega_{13}-\omega_{9} \omega_{15}\right)+b_{5}^{\prime}\left(\omega_{8} \omega_{15}-\omega_{11} \omega_{12}\right)+b_{7}^{\prime}\left(\omega_{9} \omega_{12}-\omega_{8} \omega_{13}\right)\right] \\
& m_{7}=\left(\lambda b_{2}^{\prime}-\lambda \mu b_{3}^{\prime}\right)\left[b_{4}^{\prime}\left(\omega_{9} \omega_{14}-\omega_{10} \omega_{13}\right)+b_{5}^{\prime}\left(\omega_{10} \omega_{12}-\omega_{8} \omega_{14}\right)+b_{6}^{\prime}\left(\omega_{8} \omega_{13}-\omega_{9} \omega_{12}\right)\right]
\end{aligned}
$$

Then we have the same result as in case 4.

Case 6. $\operatorname{rank}(B)=1 \neq \operatorname{rank}(B \backslash H)$. In this case the centers of the possible pseudospheres tends to a straight line at infinity. The corresponding pseudospheres degenerate and formed a pencil of hyperplanes. They contain 4 dimensional subspaces, which contains the given starting pseudohyperbolic surface $k_{\circ}$ and the corresponding velocity vectors. This leads directly to the well known result in $\mathrm{E}^{3}$, that there is in general will be no series of pseudospheres tangent to the three-dimensional surfaces.

\section{Curve of centers of the pseudospheres}

Now, we consider $t$ is varying and in this section, we will determine the centers of pseudospheres which contain a pseudohyperbolic surface $k(t)$ and are tangent to all tangent planes $\tau(t, \theta, \phi)$ of the three-dimensional surface (2). Let $a_{i}(t), i=1,2, \ldots, 7$ are the column vectors of the matrix $A(t)$, then (2) can be represented in the following way

$$
X(t, \theta, \phi)=s(t)\left[a_{1}(t) \cosh \theta+a_{2}(t) \sinh \theta \sin \phi+a_{3}(t) \sinh \theta \cos \phi\right]+d(t),
$$

where $d(t)$ is the center of the moving pseudohyperbolic surface and $a_{1}(t), a_{2}(t), a_{3}(t)$ are three orthogonal vectors in the space of the moving pseudohyperbolic surface. The velocity vectors of the points of the sphere are given by

$$
\begin{aligned}
X^{\prime}(t, \theta, \phi)= & {\left[s^{\prime}(t) a_{1}(t)+s(t) a_{1}^{\prime}(t)\right] \cosh \theta+\left[s^{\prime}(t) a_{2}(t)+s(t) a_{2}^{\prime}(t)\right] \sinh \theta \sin \phi } \\
& +\left[s^{\prime}(t) a_{3}(t)+s(t) a_{3}^{\prime}(t)\right] \sinh \theta \cos \phi+d^{\prime}(t) .
\end{aligned}
$$

The equation of the hyperplanes orthogonal to such a path is

$$
Y^{T} X^{\prime}(t, \theta, \phi)=X^{T}(t, \theta, \phi) X^{\prime}(t, \theta, \phi),
$$

where $Y=\left(y_{1}, y_{2}, y_{3}, y_{4}, y_{5}, y_{6}, y_{7}\right)^{T}$ is the position vector of an arbitrary point $Y$ in the hyperplane. The scalar product in the above equation is Lorentz metric. According to the inner product this equation is

$$
Y^{T} \varepsilon X^{\prime}(t, \theta, \phi)=X^{T}(t, \theta, \phi) \varepsilon X^{\prime}(t, \theta, \phi),
$$

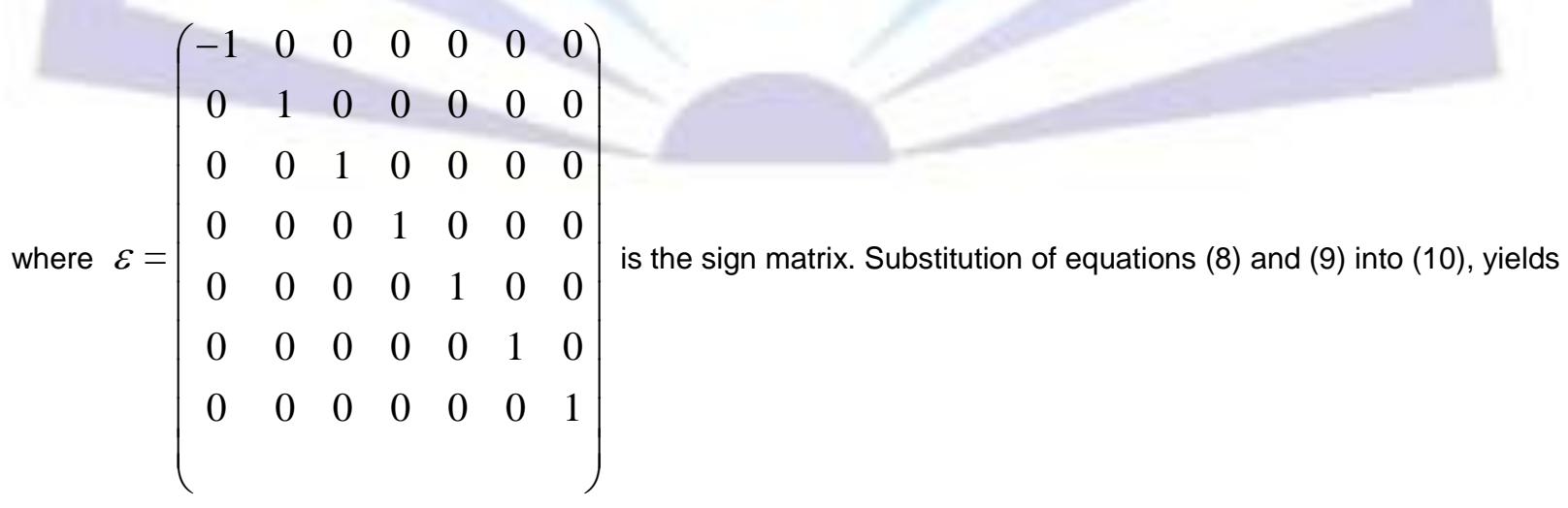




$$
\begin{aligned}
Y^{T} & \varepsilon\left[s^{\prime}(t) a_{1}(t)+s(t) a_{1}^{\prime}(t)\right] \cosh \theta+Y^{T} \varepsilon\left[s^{\prime}(t) a_{2}(t)+s(t) a_{2}^{\prime}(t)\right] \sinh \theta \sin \phi \\
& +Y^{T} \varepsilon\left[s^{\prime}(t) a_{3}(t)+s(t) a_{3}^{\prime}(t)\right] \sinh \theta \cos \phi+Y^{T} \varepsilon d^{\prime}(t) \\
& =\left(s(t) a_{1}^{T}(t) \cosh \theta+s(t) a_{2}^{T}(t) \sinh \theta \sin \phi+s(t) a_{3}^{T}(t) \sinh \theta \cos \phi+d^{T}(t)\right) \\
& \varepsilon\left(\left[s^{\prime}(t) a_{1}(t)+s(t) a_{1}^{\prime}(t)\right] \cosh \theta+\left[s^{\prime}(t) a_{2}(t)+s(t) a_{2}^{\prime}(t)\right] \sinh \theta \sin \phi\right. \\
& \left.+\left[s^{\prime}(t) a_{3}(t)+s(t) a_{3}^{\prime}(t)\right] \sinh \theta \cos \phi+d^{\prime}(t)\right) .
\end{aligned}
$$

Since $A^{T} \varepsilon A=\varepsilon$ and $A^{T} \varepsilon A^{\prime}$ is a skew symmetric matrix, let $e_{k}(t)=a_{k}^{T}(t) \varepsilon d^{\prime}(t), h_{k}(t)=a_{k}^{\prime}(t) \varepsilon d^{T}(t)$ and $\ell_{k}(t)=a_{k}(t) \varepsilon d^{T}(t), k=1,2,3$. Then by comparing the coefficients of

$\{1, \cosh \theta, \sinh \theta \sin \phi, \sinh \theta \cos \phi\}$ in (11), we obtain

$$
\begin{gathered}
\sum_{i=1}^{7} \varepsilon_{i} y_{i} b_{i}^{\prime}(t)=\sum_{i=1}^{7} \varepsilon_{i} b_{i}(t) b_{i}^{\prime}(t)-s(t) s^{\prime}(t), \\
s^{\prime}(t) \sum_{i=1}^{7} \varepsilon_{i} y_{i} a_{i 1}(t)+s(t) \sum_{i=1}^{7} \varepsilon_{i} y_{i} a_{i 1}^{\prime}(t)=s(t)\left(e_{1}(t)+h_{1}(t)\right)+s^{\prime}(t) \ell_{1}(t), \\
s^{\prime}(t) \sum_{i=1}^{7} \varepsilon_{i} y_{i} a_{i 2}(t)+s(t) \sum_{i=1}^{7} \varepsilon_{i} y_{i} a_{i 2}^{\prime}(t)=s(t)\left(e_{2}(t)+h_{2}(t)\right)+s^{\prime}(t) \ell_{2}(t), \\
s^{\prime}(t) \sum_{i=1}^{7} \varepsilon_{i} y_{i} a_{i 3}(t)+s(t) \sum_{i=1}^{7} \varepsilon_{i} y_{i} a_{i 3}^{\prime}(t)=s(t)\left(e_{3}(t)+h_{3}(t)\right)+s^{\prime}(t) \ell_{3}(t) .
\end{gathered}
$$

where $\varepsilon_{1}=-1, \varepsilon_{j}=1, j=2,3,4,5,6,7$. We know from the initial position, that the hyperplanes of the three-dimensional surfaces contain a point $m(t)$ for any $t$ and $\forall \theta, \phi$ such that $m(t)=\left(0,0,0, m_{4}(t), m_{5}(t), m_{6}(t), m_{7}(t)\right)$ is the center of this pseudosphere, then from (12), one can find

$$
F M=Q,
$$

where

$$
\begin{aligned}
& F=\left(\begin{array}{cccc}
b_{4}^{\prime}(t) & b_{5}^{\prime}(t) & b_{6}^{\prime}(t) & b_{7}^{\prime}(t) \\
s^{\prime}(t) a_{41}+s(t) a_{41}^{\prime}(t) & s^{\prime}(t) a_{51}+s(t) a_{51}^{\prime}(t) & s^{\prime}(t) a_{61}+s(t) a_{61}^{\prime}(t) & s^{\prime}(t) a_{71}+s(t) a_{71}^{\prime}(t) \\
s^{\prime}(t) a_{42}+s(t) a_{42}^{\prime}(t) & s^{\prime}(t) a_{52}+s(t) a_{52}^{\prime}(t) & s^{\prime}(t) a_{62}+s(t) a_{62}^{\prime}(t) & s^{\prime}(t) a_{72}+s(t) a_{72}^{\prime}(t) \\
s^{\prime}(t) a_{43}+s(t) a_{43}^{\prime}(t) & s^{\prime}(t) a_{53}+s(t) a_{53}^{\prime}(t) & s^{\prime}(t) a_{63}+s(t) a_{63}^{\prime}(t) & s^{\prime}(t) a_{73}+s(t) a_{73}^{\prime}(t)
\end{array}\right) \\
& M=\left(\begin{array}{c}
m_{4}(t) \\
m_{5}(t) \\
m_{6}(t) \\
m_{7}(t)
\end{array}\right) \text { and } Q=\left(\begin{array}{c}
\sum_{i=1}^{7} \varepsilon_{i} b_{i}(t) b_{i}^{\prime}(t)-s(t) s^{\prime}(t) \\
s(t)\left(e_{1}(t)+h_{1}(t)\right)+s^{\prime}(t) \ell_{1}(t) \\
s(t)\left(e_{2}(t)+h_{2}(t)\right)+s^{\prime}(t) \ell_{2}(t) \\
s(t)\left(e_{3}(t)+h_{3}(t)\right)+s^{\prime}(t) \ell_{3}(t)
\end{array}\right) .
\end{aligned}
$$

If $F$ is a regular matrix, we get

$$
M=F^{-1} Q .
$$


Therefor, the coordinates of the centers of the pseudospheres in the fixed frame at any instant $t$ are given by

$$
\left(\begin{array}{l}
M_{1} \\
M_{2} \\
M_{3} \\
M_{4} \\
M_{5} \\
M_{6} \\
M_{7}
\end{array}\right)=s(t) A(t)\left(\begin{array}{c}
0 \\
0 \\
0 \\
m_{4}(t) \\
m_{5}(t) \\
m_{6}(t) \\
m_{7}(t)
\end{array}\right)+d(t) .
$$

\section{Theorem 4.1}

\section{Example 1}

\section{References}

[1] Abdel-All, N. H. and Hamdoon, F. M. 2004. Cyclic surfaces in $E^{5}$ generated by equiform motions, J. Geom. 79 (2004) $1-11$.

[2] Bottema, O. and Roth, B. 1990. Theoretical kinematic, Dover Publications Inc., New York, 1990.

[3] Gordon, V. O. and Sement Sov, M. A. 1980. A Course in Descriptive Geometry. Mir Publishers, Moscow, 1980.

[4] Jagy, W. 1998. Sphere foliated constant mean curvature submanifolds, Rocky Mount. J. Math., 28 (1998) 983-1015.

[5] López, R. 1999. constant mean curvature hypersrfaces foliated by spheres, J. of Differential Geometry and its applications. 11 (1999), 245-256.

[6] López, R. 2001. How to use MATHEMATICA to find cyclic surfaces of constant curvature in Lorentz-Minkowski space, in: Global Differential Geometry: The Mathematical Legacy of Alfred Gray, (M. Fernández, J. Wolf, Ed.) Contemporary Mathematics, 288, A. M. S., 2001, 371-375.

[7] López, R. 2000. Maximal surfaces of Riemann type in Lorentz-Minkowski space L ${ }^{3}$, Michigan Math. J.. 47 (2000), 469-496.

[8] Nassar H. Abdel-All, Hamdoon, F. M. 2004. A geomertic characterisation of two-parameter spatial motions with many locally one-dimensional point paths, J. of Applied Math. and computation, 153 (2004) 19-25.

[9] Odehnal, B., Pottmann, H. and Wallner, J. 2006. Equiform kinematics and the geometry of line elements, Beitr. Algebra Geom., 47 (2006), 567-582.

[10] O'Neill, B. 1983. Semi-Riemannian Geometry with Application to Relativity, Academic Press, (1983).

[11] Pottmann, H. and Wallner, J. 2001. Computational Line Geometry, Springer Verlag, Heidelberg, Berlin 2001.

[12] Solouma, E. M. 2012. Local study of scalar curvature of two-dimensional surfaces obtained by the motion of circle, J. of Applied Math. and computation 219 (2012) 3385-3394. 